CARSTEN PEUST

\title{
Zur Syntax der Koordination ägyptischer Nominalphrasen
}

\section{Begriffsklärung}

Nominalphrasen können im Ägyptischen grundsätzlich auf zwei unterschiedliche Weisen koordiniert werden. Zum einen finden wir die Koordination in situ (ich benutze hier einen Begriff aus der generativen Syntax), bei der an die Stelle einer Konstituente eine Gruppe koordinierter Konstituenten eingesetzt wird. Klare Beispiele dafür finden wir im Ägyptischen etwa dann, wenn in einem Satzmuster S[ubjekt] $\mathrm{P}$ [rädikat $]$ - ([O]bjekt) in die S-Position eine koordinierte Gruppe S + S tritt:

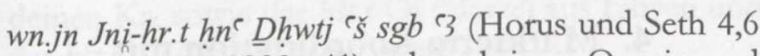
= Gardiner 1932: 41) „da schrieen Onuris und Thot laut auf"

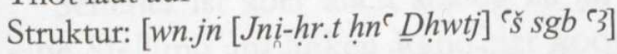

Zum anderen finden wir Koordination mit Rechtsauslagerung. Hier steht nur eines der koordinierten Glieder an seinem natürlichen Ort, während alle anderen Glieder in das Nachfeld des Satzes treten, nämlich dorthin, wo sich im Ägyptischen gewöhnlich die Adverbialien befinden:

w3.wt kkj.t 'q.t Hw jm=sn hn` Sj3 (CT VI 388 j, k), ,die dunklen Wege, auf denen $\mathrm{Hu}$ eintritt und Sia/auf denen $\mathrm{Hu}$ und Sia eintreten"

Struktur: [w3.wt kkj.t ${ }^{\mathrm{e}}$ q.t $\mathrm{Hw}$ jm $\left.=s n\right]\left[\mathrm{hn}^{\mathrm{e}} \mathrm{Sj} 3\right]$

Diese Unterscheidung ist in sehr vielen Fällen - in der Praxis bei der Masse der Belege - an der Oberfläche nicht sichtbar, obwohl sie auch hier prinzipiell vorhanden sein sollte und in der gesprochenen Sprache möglicherweise anhand der Intonation kenntlich war. Dies gilt $u$. a. für die meisten Fälle der Koordination direkter Objekte:

$j w=f h r d i . t \quad n=j p 3=j 4 q b w n$ wt $h n^{c} t 3=j \underline{d}$ db3.t n.t šs (Chonsemhab B III 5 = von Beckerath 1992: 97) „er gab mir meine vier Eingeweidekrüge und meinen Alabastersarg"
Hier könnte entweder Koordination in situ vorliegen: [jw=f $h r \underline{\text { dit.t }} n=j[p\}=j 4 q b w n$ wt $h n^{c}$ $t 3=j \underline{d b} 3 . t$ n.t $\left.\left.\check{s}^{\prime}\right]\right]$, oder aber Rechtsauslagerung: $[j w=f h r \underline{d i} . t \quad n=j p 3=j 4 q b w n \quad w t]\left[h n^{c} t 3=j \underline{d b} . t\right.$ n.t $\check{s}$ ].

Ambige Fälle dieser Art bleiben in der folgenden Diskussion unberücksichtigt. Wir wollen uns außerdem auf die Koordination satzunmittelbarer Nominalphrasen, also des Subjekts und des direkten Objekts, beschränken, und an dieser Stelle weder untersuchen, wie eingebettete (etwa von Präpositionen abhängige oder genitivische) Nominalphrasen koordiniert werden, für welche der Begriff der Rechtsauslagerung prinzipiell anders gefasst werden müsste, noch die Frage, wie andere Elemente als Nominalphrasen koordiniert werden.

\section{Unmarkierte Koordination}

Die Koordination kann im Ägyptischen bekanntlich ohne overte Markierung durch einfache Juxtaposition der Elemente erfolgen. Wir können als erste Regel konstatieren, dass die unmarkierte Koordination stets in situ konstruiert wird. Zwei Beispiele für diese sehr häufige Situation dürften genügen:

mri $R^{c} H r w$ Hw.t-Hrw fnd $=k$ pw špss (Sinuhe B $237=$ Koch 1990: 69) ,mögen Re,' Horus und Hathor diese deine ehrwürdige Nase lieben"

ž̌ Try $n$ p 3 hr hrj-pd.t hm-ntr $\check{S} d i-s w$-Hrw spr $r=j$ (LRL 30,3 = Ćerný 1939: 50) „der Nekropolenschreiber Tjaroi und der Truppenführer und Hohepriester Schedisuhor kamen zu mir“

Der einzige mir bekannte potentielle Kandidat für eine unmarkierte Koordination mit Rechtsauslagerung ist die folgende Stelle:

gmi.n $=j$ d3b.w j3rr.t jm j3q.t nb.t šps.t (Schiffbrüchiger $47 \mathrm{f} .=$ Blackman 1932: 43) 
Dies wird meist übersetzt als „Ich fand Feigen und Weintrauben dort und allerlei wertvolles Grünzeug". Ich würde eine Analyse als Apposition bevorzugen: „... (also) allerlei wertvolles Grünzeug“".

\section{Markierte Koordination mit Rechtsauslagerung: $h^{\mathrm{e}}$ und jrm}

Die Koordination kann durch verschiedene Partikeln markiert werden, unter denen die prominentesten folgende sind:

- hnc „und“ (auch „mit"; im Jüngeren Ägyptisch zunehmend durch jrm ersetzt)

- hr ,und" (auch ,auf“)

- m-mjt.t „und, sowie“ (auch „ebenso“; Varianten $r$-mjt.t und mjt.t)

- $r-p w$, oder“ (in jüngerer Sprache $m-r-p w$ ).

Die durch diese Markierungen bewirkten semantischen Schattierungen sollen hier nicht näher diskutiert werden. Nur soviel sei erwähnt, dass jeweils eine Spezialisierung eintritt gegenüber der unmarkierten Koordination, die das gesamte Spektrum „und/oder“ abdecken kann.

Die markierte Koordination geht, zumindest im Falle von $h n^{\tau}$, jrm, $h r$ und $m$-mjt.t, auf Adverbialphrasen zurück ${ }^{1}$. Die ursprüngliche Stellung dieser Phrasen muss also diejenige im Adverbialfeld des Satzes gewesen sein. Auch nach der früh erfolgten Grammatikalisierung hin zu koordinierenden Partikeln ist diese ursprüngliche Positionierung möglich geblieben und stellt, abgesehen von den weiter unten zu behandelnden Sonderfällen, die eine Konstruktion in situ erzwingen, zumindest im Älteren Ägyptisch den Normalfall dar.

Betrachten wir zunächst die Koordination mit $h n^{r}$ und jrm, so erscheint also im rechten Satzfeld die Gruppe Koordinationsmarker + Nominalphrase:

rm.w jm, hnc 3pd.w (Schiffbrüchiger 50f. = Blackman 1932: 43) „Fische gab es dort und Vögel“"

Die Etymologie von $r$-pw ist weniger klar. Fecht 1969: 110, Anm. 2, schlägt eine Herleitung aus „es ist ein Teil" vor (Hinweis von Hans-W. Fischer-Elfert). $n h h$ pw, hn $n^{e}$ d.t (CT IV 200e) „das ist die Ewigkeit und die Unendlichkeit"

jw p3j $m s-h r d d, j r m$ p3 whe (LRL 37,10 = Černý 1939: 57) „dieser Nekropolenbewohner sagte, und der Fischer"

sgnn nhb.t $=f j m, h n^{\top} q^{\top} h . w j=f j$ (pSmith 2,8 = Grapow 1958: 304) „es werde sein Hals damit gesalbt, und seine Schultern“

$j w=f m 33=f W s j r r^{c} n b, h n^{c} D h w t j$ (CT VII 507e) ,er sieht jeden Tag Osiris und Thot"

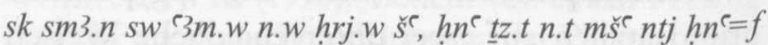
(Urk I 134,16f.), ,indes hatten ihn die asiatischen Beduinen getötet, und den bei ihm befindlichen Soldatentrupp“

$\underline{d i}=f \underline{d i}=w \quad H r w-z 3-3 s . t z 3 R^{c}-m s i n$ $m d j=f \underline{d} r=w, j r m$ rmt $n b$ j.jri mtr $r p 3$ ¿š-shn bjn Pr- 3 (Anchscheschonki 4,4f. = Glanville 1955) „er ließ Harsiese, Sohn des Ramose, in den Ofen werfen, und alle Leute, die bei ihm waren, und alle Leute, die dem Unglück des Pharao zugestimmt hatten"

\section{Markierte Koordination mit Rechtsauslagerung: $m$-mjt.t und $r$-pw}

Anders als $h n^{\top}$ und jrm stehen m-mjt.t und $r-p w$ bei Koordination mit Rechtsauslagerung in den meisten Fällen nicht vor, sondern enklitisch nach dem zu koordinierenden Nomen:

wn.jn p3 smn 'h`. (w) hr hbb3b3, d3 3 d $3=$ f m-mjt.t (pWestcar $8,21 \mathrm{f}$. = Blackman 1988: 11) „da stand die Gans auf und watschelte, und ihr Kopf ebenfalls"

jtit. $n n w y$ ph. $(w j)=f j$, šw m-mjt.t jrj (Lebensmüder $65 \mathrm{f}$. = Barta 1969: Tf. 5), die Flut nahm seinen Rest fort, und das Sonnenlicht (nahm ihn) ebenfalls (fort)"

bwpw $=k$ di jni.tw $n=j w^{c}$ dbn nbw, we dbn hd m-mjt.t (LRL 21, vs. 2f. = Černý 1939: 36) „du hast mir kein Deben Gold bringen lassen, und auch kein Deben Silber"

h3q.n=j $n^{\prime} . t j . w$ jrj $r$ Km.t, h.t=sn r-mjt.t (Urk IV 1236,6) ,ich erbeutete ihre Einwohner für Ägypten, und ihren Besitz ebenfalls“

$w t\{3\} . w d b^{r} h r=s, s 3 h r-p w$ (pHearst 11,13= Grapow 1958: 124) „es werde der Finger damit verbunden oder die Zehe"

$r \check{s} d d=k$ snf $q f n j m$, h.t jm $h z 3 r$ - $p w$ (pKahun veter. $29 \mathrm{f}$. = Grapow 1958: 547), ,so dass du geronnenes Blut dort herausholst, ,Dinge dort'(?) oder Schleim“ 
Die Partikel steht nicht, wie es nach diesen Beispielen zunächst scheint, am rechten Rand der koordinierten Nominalphrase, sondern hängt sich vielmehr enklitisch an deren erstes Element. Die folgenden Stellen zeigen, dass sie in die indirekte Genitivverbindung eintritt, wie es auch von der Kopula $p w$ her vertraut ist:

tm $m d w . t h 3 t j p w, m t . w r-p w n h 3 t j$, jnb3.(w) (pEbers 100,14f. = Grapow 1958: 8) „das bedeutet, dass das Herz nicht spricht (d.h.: schlägt) oder die Gefäße des Herzens, indem sie (vielmehr) stumm sind“

Db3.t pw n.t nb P-msn, $\underline{d} b 3=f h f t j n h m=f h r=s$, s.t $-R^{e}$ (m)-mjt.t n.t $R^{c}$ wr, hw.t-bjk n.t bjk-n-nbw.t (...) (Edfu II 9,11) „er (= der Tempel) ist das ,Edfu' des Herrn von P-msn, in dem er den Feind seiner Majestät bestraft hat, sowie der ,Sonnenthron' des großen Re, das ,Falkenhaus' des Falken-der-Goldenen, (etc.)“

$j b r, h k n w, s t j-h b \quad m j r w=f, b 3 q b 3 q\{t\} \quad m \underline{d} . t=f, n \underline{h} n m$, tw3w.t hnc md.t $n k 3=t$, h3t.t (m)-mjt.t n.t MBnw, h3t.t n.t $\check{~} \check{s}$ (Dendara IV 110,7f.) „das jbr-Öl, das hknww-Öl, ausreichend ,Festduft'-Öl, echtes helles Moringa-Öl, das $n \underline{h} n m$-Öl, das $t w 3 w . t$-Öl und das $m \underline{d} . t$-Öl sind für deinen Ka, sowie das h3t.t-Öl (Harz?) aus Libyen und das hlt.t-Öl (Harz?) von Zedern"

Seltener ist aber auch analog zu $\mathrm{hn}^{\mathrm{c}}$ und jrm eine Stellung am linken Rand der koordinierten Phrase möglich:

che.n Jni-hr.t qnd $m$ hh $n$ zp, m-mjt.t $t 3$ ps $\underline{d}$.t $r-\underline{d} r=s$ (Horus \& Seth 3,8f. $=$ Gardiner 1933: 40) „da wurde Onuris unendlich wütend, und ebenso die ganze Götterneunheit"

wnm $m s$ pnw psi.w, $r$ - $p w$ mw.t $=<f>\{k\}$ (Mutter und Kind 8,2 = Yamazaki 2003: 30) „es soll das Kind eine gekochte Maus essen, oder seine Mutter (soll eine Maus essen)“

\section{Markierte Koordination in situ}

Die markierte Koordination kann auch in situ erfolgen. Die markierte Koordination in situ ist in der älteren Sprache normalerweise wenig gebräuchlich und wird erst im Jüngeren Ägyptisch geläufiger:

jw $H w h n^{e} H k 3$ hr shr.t $n=j \underline{d} w$-qd pf (CT VII 466 b) „Hu und Heka bringen für mich jenen Bösartigen zu Fall"

$j w h m=f \underline{d} s=f h n^{\top} s n . t=f h r m h j$ (Kanopus C 8f. $=$ Urk II 130) "Seine Majestät persönlich und seine Schwester kümmerten sich" hr ptr kfi Wsr-h3.t hnc Pn-t3-wr.t $9 \mathrm{hr}$ tbn $n$ p3 jz (Turin Strike Papyrus 4, 4-6 = Gardiner 1948: 57) ,und da haben Userhat und Pentaweret Geröll über der Grabkammer abgetragen"

nn wn db3.t hn` jnr jm=f (Lefebvre 1923/4, Bd. 2: Text 81, Z. $76 \mathrm{f}$.) „es gab keinen Ziegel oder Stein (mehr) an ihm"

Anders als bei Koordination mit Rechtsauslagerung steht das Element $m$-mjt.t bei Koordination in situ offenbar stets wie $h n^{r}$ und jrm am linken Rand der von ihm angebundenen Nominalphrase (mehrere Beispiele in Abschnitt 6). Weniger gesichert ist eine entsprechende Regel für $(m)-r-p w$, da sich hier neben Belegen mit Linksstellung auch einer mit Rechtsstellung findet:

bn jrit $n 3$ kth.w hrd.w j.msi.w $n=f m-r-p w n 3$ hrd.w $n p 3=f$ $j t j d r=w r h{ }^{~} q$ h $r=w$ (Stèle de l'apanage 24f. = Menu 1998: 203,15; 22. Dyn.) „es sollen nicht die anderen Kinder, die ihm geboren wurden, oder alle die Kinder seines Vaters an ihnen teilhaben können"

jr $s d m=k$ p3 $n f r$ m-r-pw bjn (Amenemope 11,8 = Lange 1925: 60) „wenn du Gutes oder Schlechtes hörst ${ }^{6^{2}}$

Aber:

ntj $z$ z.t r-pw jm=sn (Ritner 1990: 27,11; ramessidisch) ,,in denen ein Mann oder eine Frau ist"

Kroeber 1970: 54-56, gibt die Regel, dass im Mittelägyptischen die Stellung $A B r$ - $p w$, im Neuägyptischen die Stellung $A(m)-r-p w B$ üblich sei, doch stimmt auch dies nicht völlig (vgl. z. B. den Beleg am Ende von Abschnitt 4).

Die Fälle von Koordination mit $h r$ sind syntaktisch schwer festzulegen, da $h r$ typischerweise unspezifische unzählbare und Massennomina koordiniert, die nur selten in der für unsere Fragestellung wichtigen Subjektstellung auftreten. Das folgende ist der einzige mir bekannte nichtambige Beleg; er offenbart eine Koordination in situ. Da auch sonst bekannt ist, dass $h r$ (koptisch

Während wir in Fällen wie diesem normalerweise nicht zwischen Koordination in situ und Koordination mit Rechtsauslagerung unterscheiden können, dürfte in diesem speziellen Beispiel der für die ganze koordinierte Phrase gemeinsame Artikel wohl klar auf die Struktur $p 3$ [nfr $m-r-p w b j n]$, also auf Koordination in situ, hinweisen. 
21) gern Einheiten unterhalb der Phrasenebene verbindet und sich die Koordination mit $h r$ damit als besonders eng erweist (vgl. ShishaHalevy 1986: 161, Ernst 1994: 90-93), möchte ich bis auf weiteres die Regel vorschlagen, dass die Koordination mit $h r$ grundsätzlich in situ erfolgt:

[j3]w.w hr hrd.w $m h^{\ulcorner} \cdot w t$ (Urk VII 4,2) „Alt und Jung war in Freude"

\section{Koordination im linken Satzfeld}

Es gibt gewisse Positionen im linken Satzfeld, aus denen heraus eine Auslagerung in das Adverbialfeld des Satzes nicht möglich ist und in denen daher auch die markierte Koordination durchwegs und schon seit den ältesten Zeiten in situ erfolgen muss. Dies betrifft:

(1) Die durch jn (neuägyptisch z. T. $m$ geschrieben) markierte Fokusposition in der sogenannten Cleft-Sentence:

jn $\breve{S} w h n^{r}$ Tfnw.t m3e.w tw (PT 2099 b) „Schu und Tefnut sind es, die dich führen "

jn whe $h n^{\top} w r-h r p-h m w . t h n^{\top} m d h-q d w-n z w$ jwi (Urk I 20,7) „es waren der Fischer(?) und der oberste Techniker und der königliche Architekt, die kamen"

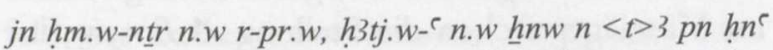
$w^{c} b$.w n.w ntr.w jrr qnb.t $n b$. $(t) n 3 b[b=s] n$ (Dekret des Haremhab $=$ Kruchten 1981: Tf. 2, D 7) ,(nur) die Propheten der Tempel, die Bürgermeister aus dem Inland und die Götterpriester sind es, die irgendeine Gerichtssitzung abhalten dürfen, die sie wünschen"

$j<n>\{r\} w^{e} q r^{e} w n h m=f m-r-p w$ w $w^{e} h r j j h w w^{e}$ smsw $n$ Pr-3 ntj j.jri=f thm (pTurin A vs. 4,1f. = Caminos 1954: 508), „es ist (nur) ein Schildträger Seiner Majestät oder ein Stallmeister (oder) ein Gefolgsmann Pharaos, der kommandieren darf"

$n t=f \underline{d} d: m r w d m n$ m-r-pw $w^{\top} w$ mn thj $r=j$ (KRI V 344 , $5 \mathrm{f}$.$) , und er soll sagen, dass der und der Inspektor$ oder der und der Soldat es sei, der ihn geschädigt habe"

Eine Auslagerung koordinierter Elemente aus $j n$-Phrasen ist gelegentlich angenommen worden, aber wohl zu Unrecht:

jn $m w t m w t h n^{c} \underline{h} r w=f$ (Bauer B $126=$ Parkinson 1991: 23)
Dies wird traditionell als Cleft-Sentence etwa in dem Sinne * ,der Sterbende ist es, der mitsamt seinem Haushalt stirbt" aufgefasst, was inhaltlich wenig befriedigt. Hier ist sicher besser im Anschluss an Quack 1992: 29, Anm. c, ein mit jn gebildeter Fragesatz im Prospektiv/Subjunktiv anzunehmen: „Soll denn ein Sterbender mitsamt seinem Haushalt sterben?"

$j m i=k \underline{d} d:$ jnk $\underline{d} d n=k n w, j n G b \underline{d} d n=k n w h n^{\ulcorner} W s j r$ (CT I $302 \mathrm{~d}-\mathrm{f}$ ) „meine nicht, dass ích dir dies sagen würde, sondern Geb ist es, der dir dies sagt, und Osiris“"

Die bisherigen Übersetzer haben $W_{s j r}$ zu $G b$ gezogen; bei Faulkner 1973/8, Bd. 1: 67, etwa lesen wir: „It is Gēb and Osiris who say this to you". Da sich aber in diesem Sargtextspruch ein Sprecher an zwei Gottheiten, nämlich Osiris und Anubis, wendet (CT I 299 d, e), ist die plausiblere Analyse ,... Geb ist es, der dir (= Anubis) dies sagt, und (auch dem) Osiris“.

(2) Das durch jr markierte Topic:

jr sn $\underline{d} . t=(j) h m-k 3 N f r-h t p h n^{c} m s . w=f n j t j m w . t, j w=s n$ $m h m-k 3$ d.t $t=(j)$ (Grdseloff 1943: 39, 2f.), was den Genossen meiner Totenstiftung, den $h m-k 3$-Priester Neferhotep, und seine Kinder von Vater und Mutter (d.h. rechtmäßig geborene?) betrifft, so sind sie die $h m-k 3$-Priester meiner Totenstiftung"

jr $n z w n b h n^{\ulcorner} n 3=w$ hm.wt-nzw mw.wt-nzw ms.w-nzw ntj htp $m$ p 3 hr 3 špsj hn ${ }^{r} n 3 n t j h t p m$ t3j s.t-nfr.w, st wd3 (pAbbott 6,5f. = Peet 1930: Tf. 3) ,,was alle Könige und ihre Königsgemahlinnen, Königsmütter und Königskinder betrifft, die in der großen ehrwürdigen Nekropole ruhen, und die im Tal der Königinnen ruhen, so sind sie unversehrt"

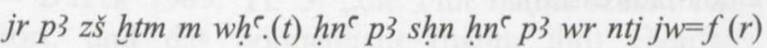
smn $t 3$ wd.t, $j w=f \underline{h} r$ hz.wt $n$ Jmnw $-R^{c}$ (Kleine DachlaStele $10-12$ = Janssen 1968: Tf. 25) ,was den siegelberechtigten Schreiber in der Oase oder den Befehlshaber oder den Würdenträger betrifft, der das Dekret einhalten wird, so soll er in der Gunst des Amun-Re sein"

jr p3 n.t- ${ }^{-}$mtj wn.w dj $m$ h3w Spll p3 wr 3 n Ht3 m-mjt.t p3 n.t- $m$ tj wn.w m h3w Mwtl p3 wr 3 n Ht3 $p 3=j$ jtj, $m h=j j m=f$ (Hethitervertrag $=$ Edel 1997: 28*) ,was den exakten Vertrag betrifft, der hier zur Zeit des hethitischen Großfürsten Suppiluliuma bestand, sowie den exakten Vertrag, der zur Zeit des hethitischen Großfürsten Muwatalli, meines Vaters, bestand, so nehme ich ihn wieder auf"

jr z3-nzw nb m-r jw $\left({ }^{c} y . t\right) n b$ rwd nb zš nb n p $3 t 3 n$ K3š ntj $j w=f r w s \underline{t n}(\ldots) m-m j t . t p 3[n] t j j w=f(r) j \underline{t} 3 w^{r} w n b(\ldots)$, 
jri.tw $h p r=f$ (KRI I 57, 2-4), was irgendeinen Vizekönig, irgendeinen Garnisonskommandanten, irgendeinen Inspektor oder irgendeinen Schreiber des Landes Nubien betrifft, der hemmungslos sein sollte (...), sowie denjenigen, der irgendeinen Matrosen fortnehmen sollte $(. .$.$) , so ist gegen ihn das Gesetz$ anzuwenden"

jr jh.t $n b .(t)$ jnk $h n^{c} t 3$ s.t qrs.t m-mjt.t s.t $n b .(t) n p 3=j$ [..] $m$-mjt.t [...] jnk, jw $=w$ n Nht-m-mw.t (oPetrie 18,8f. = Černý \& Gardiner 1957: Tf. 70,1) „was all meinen Besitz betrifft und den Begräbnisplatz sowie alle Plätze meines [...] sowie das [...] von mir, so sollen sie dem Nachtemmut gehören"

(3) Vermutlich auch gewisse auf andere Weise konstruierte Topics, etwa in der von Schenkel 2005: 82f. als „Rang IV“ bezeichneten Position. Hier wären noch Detailuntersuchungen nötig. Man betrachte folgende Beispiele:

$m=\underline{t} n \quad p r=j \quad r-d r=f \quad m-m j t . t \quad \underline{h} r d . w=j$, jnk h.t $n b .(t)$ (pHeqanachte II $25 \mathrm{f}$. = Allen 2002: Tf. 30) „seht, mein ganzer Haushalt sowie meine Kinder, alles gehört mir" (anders Allen)

ptr $\underline{t} b . w=k$ hn ${ }^{e}{ }^{\top} n . w t=k, j w=w$ mn (Roeder 1911: $\left.\int 140,2\right)$,siehe, deine Sohlen und deine Zehen, die stehen fest"

$s^{\top}{ }^{\top} w h n^{r} t 3-m h j . t, w 3 h=s n d p . w=s n m t 3$ (Hammamat Nr. 191, 9 = Couyat \& Montet 1912: 98; 11. Dyn.) „Ober- und Unterägypten, sie legen ihre Köpfe auf den Boden“

n3 j3w.t.w jrm n3 hrm.w, st šll hrr p3=t ‘̌šshn (Sonnenauge 10,27 = de Cenival 1988), „die Alten und die Jungen, sie beten für deine Sache ${ }^{\text {“ }}$

(in einer Liste:) $p 3$ twt $m$ ht $m$-mjt.t $t 3=f$ dby.t, jri.n jp.t 1 (oGardiner 3,6 = Černý \& Gardiner 1957: Tf. 22, 2) „die Statue aus Holz sowie ihr Sockel, macht eine Oipe“

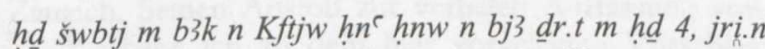
dbn 56 qd.t 3 (Urk IV 733, 5-7), ,ein silberner šwbtjKrug aus kretischer Produktion und vier Gefäße aus Erz mit Griffen aus Silber, macht 56,3 Deben“"

wh`.w nb grg.w nb ḩ3m sht 3pd.w, hn $n^{\ulcorner}$bhs $n b$ sht $m 3 j$ nb $h r$ h $3 s . t, \check{s} d i=j$ st $m r-10 m$ zbj (Hungersnotstele $25=$ Barguet 1953: Tf. 5), ,alle Fischer und alle Fallensteller, die fischen oder Vögel fangen, und alle, die in der Wüste jagen oder Löwen fangen, die besteuere ich mit einem Zehntel von der Beute"

Eine unmarkierte Koordination im linken Satzfeld kann, wie sich aus den bis hierher formulierten Regeln von selbst ergibt, ebenfalls nur in situ konstruiert werden. Für diese häufige Situation wird ein Beispiel genügen: jr jf qs.w n.w NN pn, nhn.jw pj (PT 548 b Pepi) ,was das Fleisch und die Knochen dieses NN betrifft, so sind sie jung"

\section{Bibliographie}

J.P. Allen 2002: The Heqanakht Papyri, New York

P. Barguet 1953: La stèle de la famine à Séhel, Le Caire

W. Barta 1969: Das Gespräch eines Mannes mit seinem $\mathrm{Ba}$, Berlin

J. von Beckerath 1992: Zur Geschichte von Chonsemhab und dem Geist, in Zeitschrift für Ägyptische Sprache und Altertumskunde 119: 90-107

A. M. Blackman 1932: Middle-Egyptian Stories, Bruxelles

Idem 1988: The Story of King Cheops and the Magicians, Reading

R. A. Caminos 1954: Late-Egyptian Miscellanies, London

F. de Cenival 1988: Le mythe de l'oeil du soleil, Sommerhausen

J. Černý 1939: Late Ramesside Letters, Bruxelles

J. Černý \& A. H. Gardiner 1957: Hieratic Ostraca I, Oxford

J. Couyat \& P. Montet 1912: Les inscriptions hiéroglyphiques et hiératiques du Ouâdi Hammâmât, Le Caire

CT $=$ A. de Buck 1935ff.: The Egyptian Coffin Texts, 7 Bde., Chicago

Dendara $=$ E. Chassinat 1935ff.: Le temple de Dendara, Le Caire

E. Edel 1997: Der Vertrag zwischen Ramses II. von Ägypten und Hattušili III. von Hatti, Berlin

Edfu = M. de Rochemonteix \& E. Chassinat $1984 \mathrm{ff.:}$ Le temple d'Edfu. Deuxième édition revue et corrigée par S. Cauville \& D. Devauchelle, Le Caire

R. Ernst 1994: NP-Koordination im Koptischen und Neuägyptischen, in Lingua Aegyptia 4: 89115

R. O. Faulkner 1973/8: The Ancient Egyptian Coffin Texts, Warminster

G. Fecht 1969: Der Totenbrief von $\mathrm{Nag}^{\mathrm{c}}$ ed-Deir, in Mitteilungen des Deutschen Archäologischen Instituts Kairo 24: 105-128

A. H. Gardiner 1932: Late Egyptian Stories, Bruxelles

Idem 1948: Ramesside Administrative Documents, Oxford

R. K. Glanville 1955: Catalogue of Demotic Papyri in the British Museum II: The Instructions of ${ }^{\mathrm{c}}$ Onchsheshonqy, London

H. Grapow 1958: Die medizinischen Texte in hieroglyphischer Umschreibung autographiert, Berlin 
B. Grdseloff 1943: Deux inscriptions juridiques de l'ancien empire, in Annales du Service des Antiquités de l'Egypte 42: 25-70

J. J. Janssen 1968: The Smaller Dâkhla Stela, in Journal of Egyptian Archaeology 54: 165-172

R. Koch 1990: Die Erzählung des Sinuhe, Bruxelles

$\mathrm{KRI}=\mathrm{K}$. A. Kitchen 1975 ff.: Ramesside Inscriptions, 8 Bde., Oxford

B. Kroeber 1970: Die Neuägyptizismen vor der Amarnazeit, Diss. Tübingen

J.-M. Kruchten 1981: Le decret d'Horemheb, Bruxelles

H. O. Lange 1925: Das Weisheitsbuch des Amenemope, København

G. Lefebvre 1923/4: Le tombeau de Petosiris, Le Caire

B. Menu 1998: Recherches sur l'histoire juridique, économique et sociale de l'ancienne Égypte II, Le Caire

R. B. Parkinson 1991: The Tale of the Eloquent Peasant, Oxford

T. E. Peet 1930: The Great Tomb-Robberies of the Twentieth Egyptian Dynasty, Oxford

PT $=$ K. Sethe $1908 \mathrm{ff} .:$ Die altägyptischen Pyramidentexte nach den Papierabdrücken und Photographien des Berliner Museums, 3 Bde., Leipzig

J. F. Quack 1992: Studien zur Lehre für Merikare, Wiesbaden

R. K. Ritner 1990: A Spell Against Night Terrors, in Journal of the American Research Center in Egypt 27: 25-41

G. Roeder 1911: Les temples immergés de la Nubie: Debod bis Bab Kalabsche I, Le Caire
W. Schenkel 2005: Tübinger Einführung in die klassisch-ägyptische Sprache und Schrift, Version blau, Tübingen

A. Shisha-Halevy 1986: Coptic Grammatical Categories, Roma

Urk I = K. Sethe 1933: Urkunden des Alten Reichs, 2. Aufl., Leipzig

Urk II $=$ K. Sethe 1904: Hieroglyphische Urkunden der griechisch-römischen Zeit, Leipzig

Urk IV $=$ W. Helck \& K. Sethe 1927 ff.: Urkunden der 18. Dynastie, Berlin/Leipzig

Urk VII $=$ K. Sethe 1935: Historisch-biographische Urkunden des Mittleren Reiches, Leipzig

N. Yamazaki 2003: Zaubersprüche für Mutter und Kind. Papyrus Berlin 3027, Berlin

\section{Summary}

This article discusses the coordination of subject and object noun phrases within sentences. Two types of coordination can be distinguished: coordination in situ and coordination with right-extraposition. It is argued that coordination is always constructed in situ when it is (1) not overtly marked, or (2) within a specific left-field of the sentence from where extraction to the right is impossible. In all the other cases, both constructions appear, with a prevalence towards right-extraposition in the older language. When coordination is marked, the marker stands either at the left periphery of the second coordinated element (the preferred option for coordination in situ), or it is inserted into a clitic position within the second coordinated element. 